\title{
EL LENGUAJE DE LA SOSTENIBILIDAD
}

\author{
Autora: Elena Valderrábano \\ elena.valderrabano@telefonica.com \\ Directora global de Ética Corporativa y Sostenibilidad de Telefónica
}

\section{Resumen}

La innovación sostenible se perfila como una vía para avanzar en la consecución de los Objetivos de Desarrollo Sostenible (ODS) al mismo tiempo que aporta valor a las compañías. Telefónica ya está aplicando esta fórmula con éxito aprovechando todo el potencial de los avances tecnológicos para mejorar el mundo.

Palabras clave: Telefónica; ODS; sostenibilidad; digitalización; innovación sostenible; compromiso global; impacto social positivo; impacto medioambiental positivo.

\section{The language of sustainability}

\author{
Abstract \\ Sustainable innovation is emerging as a way to advance in achieving the Sustai- \\ nable Development Goals (SDG) while providing value to companies. Telefonica
}


is already applying this formula successfully taking advantage of the potential of technological advance to improve the world.

Keywords: Telefónica; SDG; sustainability; digitalization; sustainable innovation; global commitment; positive social impact; positive environmental impact.

Fecha de recepción: 18/11/2019.

Fecha de aceptación: 20/11/2019.

\section{INTRODUCCIÓN}

Nos encontramos en un momento único, extraordinario y que posiblemente marcará la historia de la humanidad. Ni la Edad de Bronce, ni el Renacimiento, ni la llegada de la electricidad, habían supuesto una transformación comparable a la actual. El desarrollo tecnológico, además de los retos sociales y medioambientales que afectan a nuestro mundo están cambiando el paradigma y los modelos de relación entre organismos públicos, empresas y la sociedad en general. Y si hay un sector en el que esta transformación ha impactado de una forma más relevante, ese es precisamente el sector TIC (Tecnologías de la Información y Comunicación).

Desde que a finales de los años 60 se desarrollara la red de redes, que permite la interconexión descentralizada de distintos dispositivos, pasando por el lanzamiento de uno de los primeros ordenadores personales y la irrupción de la tecnología en la mayoría de los ámbitos de nuestra vida, han pasado apenas 50 años. Cinco décadas que han venido acompañadas de la aparición de modelos de negocio nunca vistos hasta el momento y la necesidad de que las compañías tradicionales fueran capaces de adaptarse a los nuevos tiempos.

En el caso concreto de Telefónica, somos una compañía que, durante casi cien años, nuestra actividad había consistido en vender un producto esencial para las personas: la comunicación. Para ello, hemos desarrollado unas capacidades tecnológicas y un capital humano únicos. Esto, unido a una firme apuesta por el I+D y el emprendimiento-a través de los programas de innovación abierta de Open Future y Wayra- ha permitido que Telefónica haya sido capaz de adaptarse a las necesidades demandadas por la sociedad y ser pioneros en el lanzamiento de nuevas tecnologías y soluciones.

Para Telefónica, la innovación es una herramienta esencial que nos permite no solo ser un jugador clave en la revolución digital, sino también ayudar a abordar los desafíos globales y crear valor para nuestros grupos de interés. Creemos que es posible invertir en un modelo de innovación, cuyas guías sean los Objetivos 
de Desarrollo Sostenible, y con la vista puesta en abordar los retos sociales y ambientales de modo que no solo contribuya al éxito económico del negocio sino también a su sostenibilidad.

\section{LOS ODS EN TELEFÓNICA}

Para hacer que la sostenibilidad sea un pilar transversal de nuestra compañía, es esencial trabajar en la transformación de nuestro negocio y focalizar los esfuerzos en:

a. Hacer nuestras soluciones más centradas en el ser humano.

b. Anticipar el impacto de nuestra actividad y tecnología en la sociedad y el medioambiente;

c. Responder a nuevos retos sociales y desafíos de la digitalización.

d. Crear una sociedad más inclusiva a través de nuestros productos y servicios, haciéndolos accesibles para no dejar a nadie atrás.

Alcanzar estas metas es mucho más sencillo si se cuenta con una guía consolidada que nos sirva de referente, y por ello consideramos que los ODS son una hoja de ruta idónea para la concienciación e integración de la sostenibilidad en nuestro negocio.

Teniendo en cuenta los objetivos marcados por la Agenda 2030, hemos elaborado un plan para impulsar iniciativas que contribuyan a alcanzar los Objetivos. Para ello, se han identificado aquellos ODS a los que tenemos más capacidad de actuación (Tabla 1, Figura 1).

Tabla 1, Figura 1. Gráfico de jerarquía de contribución de Telefónica a los ODS
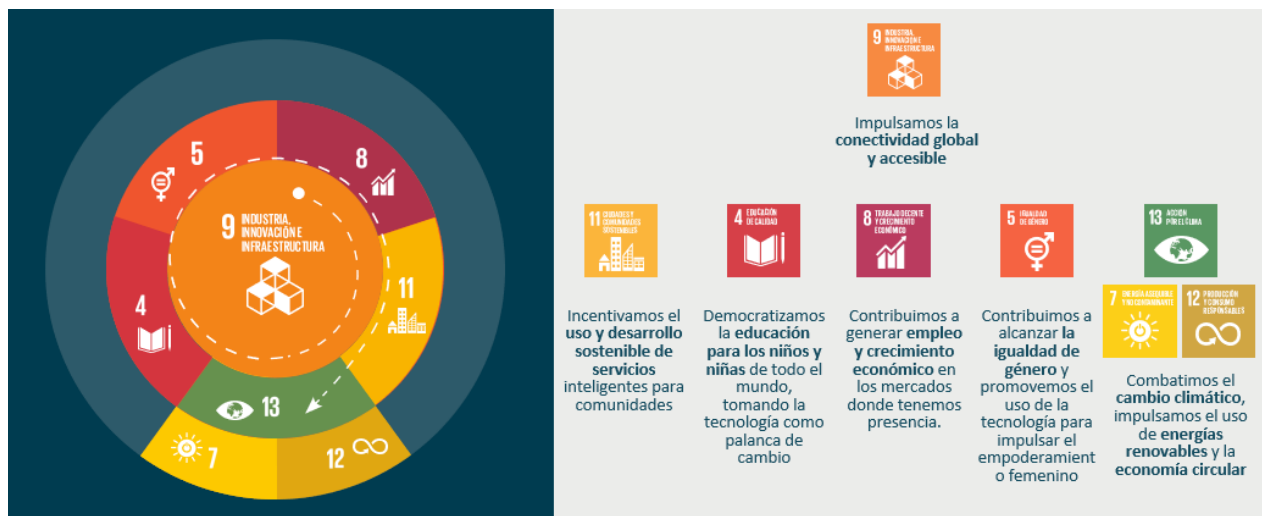

icade. Revista cuatrimestral de las Facultades de Derecho y Ciencias Económicas y Empresariales, $n^{\circ} 108$ septiembre-diciembre 2019, ISSN: 2341-0841 
En Telefónica estamos convencidos de que nuestro negocio puede contribuir de manera muy activa en la Agenda 2030 desde un doble enfoque:

a. Minimizar el impacto negativo de nuestro negocio: evolucionar y adaptar nuestras actividades comerciales, procesos y operaciones para avanzar hacia tecnologías más eficientes, reduciendo nuestro impacto en el medioambiente y garantizando los derechos humanos de nuestros empleados, clientes, accionistas y proveedores.

b. Maximizar el impacto positivo de nuestro negocio: utilizando nuestras capacidades comerciales y recursos para ofrecer nuevas tecnologías, infraestructuras y soluciones disruptivas que podrían agregar valor a las comunidades en las que operamos y permitirnos abordar la brecha digital, trayendo conectividad y servicios digitales para todos, independientemente de su ubicación, situación económica o discapacidad. Precisamente para este segundo enfoque, nos apalancamos en la llamada Innovación Sostenible que es aquella que tiene como como objetivo abordar los desafíos sociales y/o ambientales, al tiempo que creamos también un valor financiero a cambio. En definitiva, es un modelo que permite a nuestros empleados y emprendedores identificar problemas sociales y éticos, estructurar su pensamiento y adaptar sus ideas para garantizar un impacto positivo futuro mientras que garantizan la sostenibilidad del negocio.

\section{EL PROYECTO DE INNOVACIÓN SOSTENIBLE}

El proyecto de Innovación Sostenible de Telefónica se construye sobre cuatro pilares principales, a partir de los cuales se desarrollan acciones e iniciativas específicas:

a. Incorporación de la sostenibilidad al negocio a través de la capacitación y formación de los empleados y la optimización de los procesos y operaciones de la empresa.

b. Inversión en innovación con impacto, financiando soluciones innovadoras y disruptivas con un impacto social / ambiental, además de generar un modelo de negocio sostenible para la empresa.

c. Medición del impacto: seguimiento y monitoreo del impacto social / ambiental de las acciones llevadas a cabo en los ejes anteriores y su contribución a la Agenda 2030.

d. Colaboración y alianzas a través de la promoción de actividades conjuntas en colaboración con otras instituciones y asociaciones público-privadas para aumentar el impacto de las iniciativas y la inversión en nuevas soluciones y proyectos; alineado con el Objetivo 17 "Alianzas para lograr los objetivos". 


\section{PILAR 1. INCORPORACIÓN DE LA SOSTENIBILIDAD AL NEGOCIO}

Para ser sostenible, la digitalización debe ser un proceso inclusivo que garantice que todos tengamos la oportunidad de participar en un mundo conectado.

Este primer pilar se centra en la transformación interna de la empresa y sus empleados para lograr esta digitalización.

Este eje actúa sobre dos líneas de acción:

a. Capacitar y sensibilizar a todos los empleados y a todos los niveles sobre los principales desafíos identificados en la Agenda 2030 (Objetivos de Desarrollo Sostenible). Esta actividad se considera clave para garantizar un compromiso real y un cambio de cultura en la organización, para facilitar la evolución operativa que debe ser impulsada, así como para obtener el apoyo de la junta directiva de la compañía en los cambios que están por venir.

b. Optimización de los procesos y operaciones para garantizar que los proyectos, productos y servicios futuros considerarán los aspectos sociales y ambientales desde una perspectiva responsable (innovación responsable).

\section{PILAR 2. INVERTIR EN INNOVACIÓN CON IMPACTO}

A través de este segundo eje buscamos:

a. Impulsar nuevas ideas innovadoras que puedan ayudar a Telefónica a crecer como empresa, al mismo tiempo que contribuimos a los objetivos y metas de la Agenda 2030.

b. Servir como un canal que fomente el compromiso y el deseo de actuar de los empleados, de modo que no sean meros actores pasivos, sino que trabajen y propongan proyectos que aborden problemas definidos en los ODS. Como resultado, se les plantean desafíos para que presenten sus ideas o proyectos. Se financian las mejores ideas, con recursos y tutoría para llevarlas a cabo. Estas son las líneas de actuación a desarrollar en los proyectos:

- Nuevos productos y servicios o nuevas funcionalidades de soluciones existentes que tengan un impacto social / medioambiental positivo.

- Proyectos para mejorar la eficiencia interna de los procesos y del uso de recursos (reciclado, materias primas, energía...).

- Ideas que fomenten la sostenibilidad en la compañía, por ejemplo: diversidad, inclusión, DDHH, ética...

\section{PILAR 3. MEDICIÓN DEL IMPACTO}

a. Este pilar tiene como objetivo monitorizar la contribución y el impacto de nuestras actividades. Somos una de las principales fuerzas impulsoras del progreso económico en las comunidades en las que operamos. Sin embargo, 
creemos que nuestra contribución a la sociedad va más allá del valor económico que generamos para nuestros clientes y accionistas y, por lo tanto, también evaluamos y monitorizamos nuestra contribución a la sociedad y el impacto que tenemos en el medioambiente.

b. Con el objetivo de garantizar un desarrollo empresarial sostenible, hemos establecido un modelo de monitorización y medición que nos permite seguir en detalle nuestra contribución al cumplimiento de los Objetivos de Desarrollo Sostenible de las Naciones Unidas.

\section{PILAR 4. COLABORACIÓN Y ALIANZAS}

Un aspecto clave del proyecto de Innovación Sostenible de Telefónica, tanto en el trabajo de sensibilización como en la financiación de proyectos e iniciativas, es la colaboración con las instituciones para garantizar una mayor visibilidad e impacto. Estamos involucrando a las empresas privadas, el sector público y la sociedad civil en nuestro compromiso con el bienestar social y ambiental y alcanzar acuerdos con todas las partes.

Alineado con el pilar 2 (Inversión con Impacto), fomentamos la colaboración con agencias, empresas e instituciones públicas y privadas que ayudan a promover el desarrollo tecnológico financiando proyectos innovadores que tienen como objetivo abordar los desafíos sociales / ambientales.

En Telefónica creemos que a través de alianzas es posible hacer una mayor contribución para lograr un mundo más sostenible y socialmente responsable (ODS 17). Es muy importante contar con aliados tanto internos, que ayudan a conseguir que la sostenibilidad permee en más entornos de la compañía, así como externos que nos ayudan a expandir el impacto del programa y la sensibilización con la sostenibilidad y la Agenda 2030.

\section{EN CONCLUSIÓN}

Pero mucho más allá de estos pilares sobre los cuales se articulan infinidad de iniciativas con un impacto global y transversal, ya que como empleados y partícipes de la sociedad no debemos olvidar nuestro compromiso para con los demás.

Estamos viviendo un cambio de era con irrupciones únicas. Como sociedad, debemos aprovecharlo para hacer que nuestro compromiso nos ayude a salvaguardar nuestro patrimonio, entorno, cultura y bienestar. Los ODS son una excelente hoja de ruta para conseguirlo.

Nunca antes tantos países, personas y empresas habían hablado un lenguaje común y con un único fin: hacer que nuestro mundo sea inclusivo y sostenible. Este es el lenguaje de la sostenibilidad. 